\title{
SINGULAR STRESS CONCENTRATIONS IN SECOND-GRADE MATERIALS*
}

\author{
BY \\ R. W. LARDNER \\ Simon Fraser University, Burnaby, B.C., Canada.
}

\begin{abstract}
The problems of a second-grade elastic half-space deformed in plane strain and subject to a concentrated load, a discontinuous load, and a double force, acting at a point on the free surface are solved. The nature of the stress singularity is found in each case. Although the strengths of the stress singularities are the same as for both classical elasticity and conventional couple-stress materials, the detailed forms are different and depend on a certain combination of the five new elastic constants. The displacement field produced by a point load is bounded in the neighbourhood of the point of application of the force, in contrast to both earlier theories where it is singular.

1. Introduction. One of the problems of current concern in theories of continuum mechanics is to what extent it is necessary to include details of the microstructure of materials. In the classical theories of continua the basic assumption which eliminates such details is that the action upon one another of two neighbouring parts of a body consists solely of a force localised on the surface element separating the two parts. The usual elementary argument used to support such an assumption is that, for a small enough surface element, the micro-forces acting across it will all be parallel and hence will lead to no resultant couple. From an atomistic viewpoint this argument is clearly invalid: although it seems likely that the resultant couple due to all the interatomic forces will be small, it is quite unlikely that it will actually be zero. Thus it is reasonable to demand that theories of deformable media should include consideration of couple stresses.

The first attempt to include couple stresses was given in a series of papers by Grioli [1], Toupin [2], Mindlin and Tiersten [3] and Koiter [4]. It was later realised that the materials considered by these authors had certain anomalous features, and Toupin [5] pointed out that the fundamental constitutive postulate which had been made was too restrictive. It turns out that these materials are special cases of a wider class of so-called secondgrade materials, in which the constitutive assumption is that the strain energy density depends on the strain and strain gradient tensors. Such materials do not have the anomalous properties of the earlier ones. Because of this, the present author has given [6] a linearised version of the equations obeyed by second-grade elastic bodies, and has solved various vibration problems for them.

In both of the linearised couple-stress theories it is necessary to introduce a number of new elastic constants (two in the Mindlin-Tiersten case, five in the present one), and these can be regarded as introducing certain fundamental lengths. Because the classical theories of elasticity agree very well in their predictions with experimental results, it has to be concluded that these lengths are rather small. This means that the results obtained from couple-stress theories differ from those of classical elasticity by significant amounts only within a thin boundary layer close to any free surface or
\end{abstract}

${ }^{*}$ Received February 11, 1969; revised version received March 12, 1969. 
interface or else in situations where there is a small geometric length [3], [7]. Some of the results among this second group of problems offer a possibility of devising experimental tests for measuring the new elastic constants.

An extreme case of a small geometric length arises in problems with singular stress concentrations, where there is a zero length involved. Several such problems have been investigated by Muki and Sternberg [8] for the old couple-stress theory, and results were found which differed greatly from the classical results. Perhaps even more significant is the fact that the couple-stress results do not tend to the classical results as the fundamental lengths tend to zero. In view of this, it seems worthwhile extending these results to the general second-grade case.

After summarizing the equations satisfied by second-grade materials in Sec. 2, we discuss plane strain deformation in Sec. 3, and further specialise to consider a half-space in Sec. 4. It is convenient for plane strain problems to use two displacement potentials, in terms of which the equations of motion are particularly tractable. As usual for halfspace problems we use an appropriate Fourier transform, and the transforms of the potentials are found. These are then used in the boundary conditions on the free surface of the half-space, giving four equations to be solved for the unknown coefficients. We are interested in cases in which there are stress singularities at the origin, due either to a point load or to a discontinuous load or to a double force there. Consequently the asymptotic behaviour of the coefficients in the transform variable is needed, rather than the full solution.

The singularities in the three cases of classical elasticity, Mindlin-Tiersten theory and second-grade materials are compared. The strengths of the singularities are with one exception the same, but their detailed form changes from one theory to another. However, the results for all three types of material can be encompassed by a single formula for each singular stress component involving a certain constant, $k$. For classical elasticity $k=1$, for the old couple stress theory $k=(3-2 \nu)^{-1}$ where $\nu$ is Poisson's ratio, while for second-grade theory $k$ depends in a complicated way on the five new elastic constants, but not on l'oisson's ratio. Although the three theories form a hierarchy with each a special case of the next, in no case can the nature of one singularity be derived as a limiting case of another.

The displacement field produced by a point load has a logarithmic singularity in both the classical and old couple-stress theories, and this implies that an infinite strain energy is contained within the region around the point of application of the load. This is completely different from the situation in second-grade materials, where the displacement field is bounded near the point force. 'The strain energy density is singular as $(\ln r)^{2}$, and hence a finite strain energy is contained within any finite portion of the body.

The exception to the uniformity of stress singularity strengths concerns the discontinuous normal load. In the classical case all stress components are bounded near the discontinuity, while both couple stress theories show a logarithmic singularity in shear stress. (Here, as elsewhere, the singularity depends on which stress tensor we are using.)

2. Basic equations for linear second-grade materials. We consider a continuous body in which the particles have positions $\mathbf{x}$ with components $\left(x_{\alpha}\right)$ in a certain Cartesian coordinate frame, and we denote by $\left\{u_{\alpha}(\mathbf{x}, t)\right\}$ the components of the displacement of each particle at time $t$ from its position in the unstressed state of the body. In the linear approximation it is assumed that the first and second gradients of the displacement, $u_{\alpha, \beta}$ and $u_{\alpha, \beta \gamma}$, are small. If it is supposed that the material has the usual kinetic energy 
density, $\rho(\mathbf{x}) \dot{\mathfrak{u}}^{2} / 2$, and a strain energy density which is a function of the first and second displacement gradients, then it can be shown that the equations of motion can be written as [6]

$$
\rho \ddot{u}_{\alpha}=T_{\alpha \beta, \beta}+F_{\alpha}-C_{\alpha \beta, \beta},
$$

and that the boundary conditions are

$$
T_{\alpha}-T_{\alpha \beta} N_{\beta}+C_{\alpha \beta} N_{\beta}-D_{\beta}\left(D_{\alpha \beta}-M_{\alpha \beta \gamma} N_{\gamma}\right)=0
$$

and

$$
M_{\alpha \beta \gamma} N_{\beta} N_{\gamma}=D_{\alpha \beta} N_{\beta} .
$$

In these equations, $\rho$ is the density, $F_{\alpha}$ an external body force and $C_{\alpha \beta}$ a body dipolar force, $T_{\alpha}$ is the surface traction and $D_{i \alpha}$ is a surface dipolar traction. $T_{\alpha \beta}$ and $M_{\alpha \beta \gamma}$ are the stress and hyper-stress tensors, which will be defined below, $N_{\alpha}$ is the unit outward normal at the surface of the body, and $D_{\beta}$ is a surface differentiation operator defined by

$$
D_{\beta}=\frac{\partial}{\partial x_{\beta}}-N_{\beta} N_{\gamma} \frac{\partial}{\partial x_{\gamma}} .
$$

If we define the usual strain tensor

$$
e_{\alpha \beta}=\frac{1}{2}\left(u_{\alpha, \beta}+u_{\beta, \alpha}\right)
$$

then the most general quadratic form for the strain energy density $W$ in an isotropic and centro-symmetric material is

$$
\begin{aligned}
W=W_{0}+\frac{1}{2} \lambda\left(e_{\alpha \alpha}\right)^{2}+\mu e_{\alpha \beta} e_{\alpha \beta}+ & F_{1} e_{\alpha \alpha, \gamma} e_{\beta \beta, \gamma}+F_{2} e_{\alpha \alpha, \beta} e_{\beta \gamma, \gamma} \\
& +F_{3} e_{\alpha \beta, \beta} e_{\alpha \gamma, \gamma}+F_{4} e_{\alpha \beta, \gamma} e_{\alpha \beta, \gamma}+F_{5} e_{\alpha \beta, \gamma} e_{\alpha \gamma, \beta},
\end{aligned}
$$

where $\lambda, \mu, F_{1}, \cdots, F_{5}$ are elastic constants. From this we obtain the following expressions for the stress tensors (using $\Delta=u_{\alpha, \alpha}$ ):

$$
\begin{array}{r}
T_{\kappa \lambda}=\lambda \Delta \delta_{\kappa \lambda}+\mu\left(u_{\kappa, \lambda}+u_{\lambda, \kappa}\right)-\left\{\left(2 F_{1}+F_{2}\right) \delta_{\kappa \lambda} \nabla^{2} \Delta+\left(F_{2}+F_{3}+F_{5}\right) \Delta_{, \kappa \lambda}\right. \\
\left.+\left(\frac{1}{2} F_{3}+F_{4}+\frac{1}{2} F_{5}\right) \nabla^{2}\left(u_{\kappa, \lambda}+u_{\lambda, \kappa}\right)\right\} \\
M_{\kappa \lambda \mu}=2 F_{1} \delta_{\kappa \lambda} u_{\beta, \beta \mu}+\frac{1}{2} F_{2} \delta_{\kappa \lambda}\left(u_{\mu, \beta \beta}+u_{\beta, \beta \mu}\right)+\frac{1}{2}\left(F_{2}+F_{3}\right)\left(\delta_{\lambda \mu} u_{\beta, \beta \kappa}+\delta_{\kappa \mu} u_{\beta, \beta \lambda}\right) \\
+\frac{1}{2} F_{3}\left(\delta_{\lambda \mu} u_{\kappa, \beta \beta}+\delta_{\kappa \mu} u_{\lambda, \beta \beta}\right)+\left(F_{4}+\frac{1}{2} F_{5}\right)\left(u_{\kappa, \lambda \mu}+u_{\lambda, \kappa \mu}\right)+F_{5} u_{\mu, \kappa \lambda} .
\end{array}
$$

We can substitute from Eq. (7) into Eq. (1) and obtain the analogue of Navier's equations of motion in terms of the displacement field. For the case of equilibrium with no body forces these take the form

$$
(\lambda+\mu) \Delta_{, \alpha}+\mu \nabla^{2} u_{\alpha}-(\lambda+2 \mu) l_{1}^{2} \nabla^{2} \Delta_{, \alpha}-\mu l_{2}^{2}\left(\nabla^{4} u_{\alpha}-\nabla^{2} \Delta_{, \alpha}\right)=0,
$$

where

$$
(\lambda+2 \mu) l_{1}^{2}=2\left(F_{1}+F_{2}+F_{3}+F_{4}+F_{5}\right) \quad \text { and } \quad \mu l_{2}^{2}=\frac{1}{2} F_{3}+F_{4}+\frac{1}{2} F_{5} .
$$

The positive definiteness of the strain energy density implies that both $l_{1}^{2}$ and $l_{2}^{2}$ are positive.

3. Plane strain. We now restrict to the case of plane strain by assuming that $u_{3}=0$ and that all quantities are independent of $x_{3}$. Then it is convenient to introduce a pair 
of displacement potentials $\varphi\left(x_{1}, x_{2}\right)$ and $\psi\left(x_{1}, x_{2}\right)$ which can always be found such that

$$
u_{1}=\varphi_{.1}+\psi_{.2} \quad u_{2}=\varphi_{.2}-\psi_{, 1} .
$$

If we now define

$$
\Phi=(\lambda+2 \mu) \nabla^{2}\left(\varphi-l_{1}^{2} \nabla^{2} \varphi\right), \quad \Psi=\mu \nabla^{2}\left(\psi-l_{2}^{2} \nabla^{2} \psi\right),
$$

then the equilibrium equations (9) become

$$
\begin{aligned}
& \alpha=1: \Phi_{, 1}+\Psi_{, 2}=0, \\
& \alpha=2: \Phi_{, 2}-\Psi_{, 1}=0 .
\end{aligned}
$$

Consequently the problem is solved if we can find the two conjugate harmonic functions, $\Phi$ and $\Psi$. Eqs. (12) give $\varphi$ and $\psi$, from which we can obtain the displacements and hence, via Eqs. ( 7 ) and (8), the stresses.

It will be convenient later to have expressions for the stresses and some of the hyperstresses in terms of the potentials. These are

$$
\begin{aligned}
T_{11} & =\Phi-2 \mu \varphi_{, 22}+2 R \nabla^{2} \varphi_{.22}+2 \mu\left(\psi-l_{2}^{2} \nabla^{2} \psi\right)_{, 12}, \\
T_{22} & =\Phi-2 \mu \varphi_{, 11}+2 R \nabla^{2} \varphi_{.11}-2 \mu\left(\psi-l_{2}^{2} \nabla^{2} \psi\right)_{, 12}, \\
T_{12} & =2 \mu \varphi_{, 12}-2 R \nabla^{2} \varphi_{, 12}+\mu\left(\partial_{22}-\partial_{11}\right)\left(\psi-l_{2}^{2} \nabla^{2} \psi\right), \\
T_{33} & =\lambda \nabla^{2} \varphi-(P-2 R) \nabla^{4} \varphi \\
M_{111} & =P \nabla^{2} \varphi_{, 1}-2 T \varphi_{, 122}+R \nabla^{2} \psi_{, 2}+T\left(\partial_{11}-\partial_{22}\right) \psi_{, 2}, \\
M_{211} & =M_{121}=R \nabla^{2} \varphi_{, 2}+T\left(\partial_{11}-\partial_{22}\right) \varphi_{, 2}-Q \nabla^{2} \psi_{, 1}+2 T \psi_{, 122}, \\
M_{221} & =(P-2 R) \nabla^{2} \varphi_{, 1}+2 T \varphi_{, 221}+(R-2 Q) \nabla^{2} \psi_{, 2}+T\left(\partial_{22}-\partial_{11}\right) \psi_{, 2} .
\end{aligned}
$$

Here we use $\partial_{\kappa \lambda} \equiv \partial^{2} / \partial x_{\kappa} \partial x_{\lambda}$ and the following abbreviations:

$$
\begin{aligned}
& P=2\left(F_{1}+F_{2}+F_{3}+F_{4}+F_{5}\right)=(\lambda+2 \mu) l_{1}^{2}, \\
& Q=\frac{1}{2} F_{3}+F_{4}+\frac{1}{2} F_{5}=\mu l_{2}^{2}, \\
& R=\frac{1}{2} F_{2}+F_{3}+F_{4}+F_{5}, \\
& T=F_{4}+F_{5} .
\end{aligned}
$$

4. Half-space problems. We consider now the problem of a half-space $(x>0)$ subject to given tractions on its free surface, with the displacements tending to zero at infinity. Then as usual with such problems we express all quantities in terms of their Fourier transforms with respect to $y$ :

$$
\bar{f}(x, s)=\int_{-\infty}^{\infty} f(x, y) e^{i s y} d y .
$$

Since $f(x, y)$ will always be real, $\bar{f}(x,-s)=\bar{f}(x, s)^{*}$, and the inversion formula can be written

$$
f(x, y)=\frac{1}{2 \pi} \int_{0}^{\infty}\left\{\left[\bar{f}(x, s)+\bar{f}(x, s)^{*}\right] \cos s y+\frac{1}{i}\left[\bar{f}(x, s)-\bar{f}(x, s)^{*}\right] \sin s y\right\} d s .
$$


Eqs. (13) in terms of the transformed quantities become

$$
\partial \bar{\Phi} / \partial x-i s \bar{\Psi}=0, \quad \partial \bar{\Psi} / \partial x+i s \bar{\Phi}=0,
$$

and using that $\Phi$ and $\bar{\Psi}$ tend to zero as $x \rightarrow \infty$, the solutions are

$$
\Phi(x, s)=A(s) e^{-s x}, \quad \bar{\Psi}(x, s)=i A(s) e^{-s x} .
$$

Then Eq. $\left(12_{1}\right)$ becomes

$$
(\lambda+2 \mu)\left(\partial^{2} / \partial x^{2}-s^{2}\right)\left[1-l_{1}^{2}\left(\partial^{2} / \partial x^{2}-s^{2}\right)\right] \bar{\varphi}(x, s)=A(s) e^{-s x},
$$

and integrating we obtain that

$$
\bar{\varphi}(x, s)=a_{1}(s) e^{-s x}+a_{2}(s) \exp \left[-\left(s^{2}+l_{1}^{-2}\right)^{1 / 2} x\right]-\frac{A(s)}{2(\lambda+2 \mu) s} x e^{-s x} .
$$

Similarly from Eq. $\left(12_{2}\right)$

$$
\bar{\psi}(x, s)=b_{1}(s) e^{-s x}+b_{2}(s) \exp \left[-\left(s^{2}+l_{2}^{-2}\right)^{1 / 2} x\right]-\frac{i A(s)}{2 \mu s} x e^{-s x} .
$$

The boundary conditions (2) and (3) on the plane $x=0$ take a simple form. In this case $\mathbf{N}=(-1,0,0)$ and the surface differentiation operator is $\left(D_{\alpha}\right)=\left(0, \partial_{2}, \partial_{3}\right)=$ $\left(0, \partial_{2}, 0\right)$. Then Eqs. (2) and (3) become

$$
\begin{aligned}
& T_{11}-\partial_{2}\left(M_{121}\right)=-T_{1}, \\
& T_{21}-\partial_{2}\left(M_{221}\right)=-T_{2}, \text { on } x=0, \\
& M_{111}=-D_{11}, \\
& M_{211}=-D_{21} .
\end{aligned}
$$

We shall consider problems in which no dipolar tractions are applied, so that the righthand sides of the last two equations are zero and the second term in the first equation is absent. Then the transforms of the resulting boundary conditions are

$$
\begin{aligned}
\bar{T}_{11} & =-\bar{T}_{1}, \quad \bar{T}_{21}+i s \bar{M}_{221}=-\bar{T}_{2}, \quad \text { on } x=0, \\
\bar{M}_{111} & =\bar{M}_{211}=0 .
\end{aligned}
$$

Now we substitute the representations (24)-(26) for the potentials into Eqs. (14)-(20) to obtain the stresses, and substitute these in turn into the boundary conditions (28). The resulting equations are

$$
\begin{aligned}
-\left.\bar{M}_{111}\right|_{x=0}= & 2 T s^{3}\left(a_{1}+i b_{1}\right)+a_{2}\left(s^{2}+l_{1}^{-2}\right)^{1 / 2}\left[\lambda+2 \mu+2 T s^{2}\right] \\
& \quad+i s b_{2}\left[(R+T) l_{2}^{-2}+2 T s^{2}\right]+s A(s)\left[\frac{P+T}{\lambda+2 \mu}-\frac{R+T}{\mu}\right]=0 \\
\left.i \bar{M}_{211}\right|_{x=0}=2 T s^{3}\left(a_{1}+i b_{1}\right)+a_{2} s\left[(R+T) l_{1}^{-2}+2 T s^{2}\right] & \\
& +i b_{2}\left(s^{2}+l_{2}^{-2}\right)^{1 / 2}\left[\mu+2 T s^{2}\right]+s A(s)\left[\frac{R+T}{\lambda+2 \mu}-\frac{Q+T}{\mu}\right]=0
\end{aligned}
$$




$$
\begin{gathered}
\left.\bar{T}_{11}\right|_{x=0}=2 \mu s^{2}\left(a_{1}+i b_{1}\right)+2 s^{2} a_{2}\left[\mu-R l_{1}^{-2}\right]+2 s^{2} A(s)\left[\frac{Q}{\mu}-\frac{R}{\lambda+2 \mu}\right]=-\bar{T}_{1} ; \\
-\left.i\left(\bar{T}_{12}+i s \bar{M}_{221}\right)\right|_{x=0}=s^{2}\left(a_{1}+i b_{1}\right)\left[2 \mu+2 T s^{2}\right] \\
+a_{2} s\left(s^{2}+l_{1}^{-2}\right)^{1 / 2}\left[-\lambda+2 T s^{2}\right]+i b_{2} s^{2}\left[2 \mu+(T-R) l_{2}^{-2}+2 T s^{2}\right] \\
+A(s)\left[-\frac{\lambda+\mu}{\lambda+2 \mu}+s^{2}\left(\frac{T-P}{\lambda+2 \mu}+\frac{R-T}{\mu}\right)\right]=i \bar{T}_{2} .
\end{gathered}
$$

It is a straightforward, if laborious, job to obtain the coefficient functions $\left(a_{1}+i b_{1}\right)$, $a_{2}, i b_{2}$ and $A$ from these equations and then to use them to find the stress and hyperstress transforms. In the stresses, obtained by combining Eqs. (14)-(16) and (24)-(26), it is convenient to use Eq. (31) to eliminate the coefficient $a_{2}$, and we then obtain

$$
\begin{gathered}
\bar{T}_{11}(x, s)=e^{-s x}\left\{2 \mu s^{2}\left(a_{1}+i b_{1}\right)\left(1-e^{-\kappa x}\right)-\bar{T}_{1} e^{-\kappa x}\right. \\
\left.+A(s)\left[\frac{\lambda+\mu}{\lambda+2 \mu} s x+2 s^{2}\left(\frac{Q}{\mu}-\frac{R}{\lambda+2 \mu}\right)\left(1-e^{-\kappa x}\right)\right]\right\} \\
\bar{T}_{12}(x, s)=i e^{-s x}\left\{2 \mu s^{2}\left(a_{1}+i b_{1}\right)\left[1-\left(1+\frac{1}{s^{2} l_{1}^{2}}\right)^{1 / 2} e^{-\kappa x}\right]-\bar{T}_{1}\left(1+\frac{1}{s^{2} l_{1}^{2}}\right)^{1 / 2} e^{-\kappa x}\right. \\
\left.+A(s)\left[\frac{\lambda+\mu}{\lambda+2 \mu}(s x-1)+2 s^{2}\left(\frac{Q}{\mu}-\frac{R}{\lambda+2 \mu}\right)\left(1-\left(1+\frac{1}{s^{2} l_{1}^{2}}\right)^{1 / 2} e^{-\kappa x}\right)\right]\right\} \\
\bar{T}_{22}(x, s)=-e^{-s x}\left\{2 \mu s^{2}\left(a_{1}+i b_{1}\right)\left[1-\left(1+\frac{1}{s^{2} l_{1}^{2}}\right) e^{-\kappa x}\right]-\bar{T}_{1}\left(1+\frac{1}{s^{2} l_{1}^{2}}\right) e^{-\kappa x}\right. \\
\left.+A(s)\left[\frac{\lambda+\mu}{\lambda+2 \mu}(s x-2)+2 s^{2}\left(\frac{Q}{\mu}-\frac{R}{\lambda+2 \mu}\right)\left(1-\left(1+\frac{1}{s^{2} l_{1}^{2}}\right) e^{-\kappa x}\right)\right]\right\},
\end{gathered}
$$

where $\kappa=\left(s^{2}+l_{1}^{-2}\right)^{1 / 2}-s$. In a similar way, expressions can be obtained for the hyperstresses. This enables us to solve an arbitrary stress boundary value problem for a half-space, at least up to a Fourier inversion.

We shall also be interested in the displacements whose transforms can be obtained from Eqs. (11), (25) and (26). After using Eq. (29) to remove the terms in $\left(a_{1}+i b_{1}\right)$ we obtain

$$
\begin{gathered}
\bar{u}_{1}(x, s)=e^{-s x}\left\{a_{2}\left(s^{2}+l_{1}^{-2}\right)^{1 / 2}\left[\frac{\lambda+2 \mu}{2 T s^{2}}+\left(1-e^{-\kappa x}\right)\right]\right. \\
\left.+i b_{2} s\left[\frac{(R+T) l_{2}^{-2}}{2 T s^{2}}+\left(1-e^{-\omega x}\right)\right]+\frac{A(s)}{2 T s}\left[\frac{P+T s x}{\lambda+2 \mu}-\frac{R+T+T s x}{\mu}\right]\right\} \\
\bar{u}_{2}(x, s)=i e^{-s x}\left\{a_{2}\left[\left(s^{2}+l_{1}^{-2}\right)^{1 / 2} \frac{\lambda+2 \mu}{2 T s^{2}}+\kappa+s\left(1-e^{-\kappa x}\right)\right]\right. \\
+i b_{2}\left[\frac{(R+T) l_{2}^{-2}}{2 T s}-\omega+\left(s^{2}+l_{2}^{-2}\right)^{1 / 2}\left(1-e^{-\omega x}\right)\right] \\
\left.+\frac{A(s)}{2 T s}\left[\frac{P+T+T s x}{\lambda+2 \mu}-\frac{R+T s x}{\mu}\right]\right\} .
\end{gathered}
$$

Here $\omega=\left(s^{2}+l_{2}^{-2}\right)^{1 / 2}-s$.

In the next section we shall look at the particular case of concentrated loads. 
5. Stress singularities of point loads. We consider separately the cases of normal and shear loadings which are concentrated in the neighbourhood of the origin. In the limit of point loads we have:

Case A

$$
\begin{array}{lll}
T_{1}(y)=\Pi \delta(y), & \bar{T}_{1}(s)=\Pi, & \bar{T}_{2}(s)=0 ; \\
T_{2}(y)=\Sigma \delta(y), & \bar{T}_{2}(s)=\Sigma, & \bar{T}_{1}(s)=0 .
\end{array}
$$

Case B

We are interested in the nature of the singular part of the stress field in the neighbourhood of the point force. Consequently we do not need to solve Eqs. (29)-(32) completely, but merely for the asymptotic limits of the coefficient functions as $s \rightarrow \infty$. However, even with this limited goal we cannot succeed without considerable algebraic effort since, as can readily be seen, the rank of the leading terms in the four equations is only two. This rank is increased to four if the terms in $\left(a_{1}+i b_{1}\right)$ are first eliminated from Eqs. (29), (30) and (32).

As $s \rightarrow \infty$ the coefficients have the forms

$$
\begin{gathered}
a_{2}(s) \sim l_{1}^{2} a+a^{\prime} / s^{2} \cdots, \quad i b_{2}(s) \sim l_{2}^{2} b+b^{\prime} / s^{2}+\cdots, \\
a_{1}(s)+i b_{1}(s) \sim \gamma+\gamma^{\prime} / s^{2} \cdots, \quad A(s) \sim A_{1}+A_{2} / s^{2} \cdots .
\end{gathered}
$$

Then, having performed the elimination mentioned above, the leading terms in the four equations are:

$$
\begin{aligned}
\mu \gamma+\left(l_{1}^{2} \mu-R\right) a+\left(\frac{Q}{\mu}-\frac{R}{\lambda+2 \mu}\right) A_{1} & =0, \\
(R-P) a+(Q-R) b-\left(\frac{Q-R}{\mu}+\frac{P-R}{\lambda+2 \mu} A_{1}\right) & =0, \\
R a+Q b+\left(\frac{R}{\lambda+2 \mu}-\frac{Q}{\mu}\right) A_{1} & =0, \\
\left(\mu-T / 2 l_{1}^{2}\right) a-\left(\mu-T / 2 l_{2}^{2}\right) b-\frac{\lambda+\mu}{\lambda+2 \mu} A_{1} & =\bar{T}_{1}+i \bar{T}_{2} .
\end{aligned}
$$

Provided the material is such that

$$
\left[P Q-R^{2}\right][4 P Q-T(P+Q)] \neq 0,
$$

the solution of these equations is:

$$
\begin{aligned}
b & =A_{1} / \mu, \quad a=-A_{1} /(\lambda+2 \mu), \\
\gamma & =\left[\frac{P}{(\lambda+2 \mu)^{2}}-\frac{Q}{\mu^{2}}\right] A_{1} \text { and } A_{1}=-\frac{2 P Q\left(\bar{T}_{1}+i \bar{T}_{2}\right)}{4 P Q-T(P+Q)} .
\end{aligned}
$$

Returning to the stresses (Eqs. (33)-(35)), as $s \rightarrow \infty$ they have limiting form

$$
\begin{aligned}
& \bar{T}_{11}(x, s) \sim e^{-s x}\left\{-\bar{T}_{1}+K s x\right\}, \\
& \bar{T}_{12}(x, s) \sim i e^{-s x}\left\{-\bar{T}_{1}+K(s x-1)\right\}, \\
& \bar{T}_{22}(x, s) \sim-e^{-s x}\left\{-\bar{T}_{1}+K(s x-2)\right\},
\end{aligned}
$$

where

$$
K=\frac{\mu}{l_{1}^{2}} \gamma+A_{1}\left[\frac{l_{2}^{2}}{l_{1}^{2}}-\frac{R}{P}+\frac{\lambda+\mu}{\lambda+2 \mu}\right] .
$$


The terms not included in Eqs. (44) are proportional at most either to $x s^{-1}$ or to $s^{-2}$, and both of these lead to terms in the stresses which are bounded near the origin. Finally, using Eq. (43) we obtain that

$$
K=-k\left(T_{1}+i T_{2}\right) \quad \text { where } \quad k=2 Q(P-R) /(4 P Q-T(P+Q)) .
$$

In order to find the limiting behaviour of the stresses themselves we make use of the following results [8]:

$$
\begin{array}{ll}
\int_{0}^{\infty} e^{-s x} \cos s y d s \sim \frac{2 x}{r^{2}}, & \int_{0}^{\infty} s e^{-s x} \cos s y d s \sim \frac{2\left(x^{2}-y^{2}\right)}{r^{4}} \\
\int_{0}^{\infty} e^{-s x} \sin s y d s \sim \frac{2 y}{r^{2}}, & \int_{0}^{\infty} s e^{-s x} \cos s y d s \sim \frac{2 x y}{r^{4}}
\end{array}
$$

as $r=\left(x^{2}+y^{2}\right)^{1 / 2} \rightarrow 0$. Then combining the asymptotic forms (44) with the inversion formula (23) for the two particular cases of Eq. (37) gives the following singular behaviours as $r \rightarrow 0$ :

Case A

$$
\begin{aligned}
& T_{11} \sim-\frac{\Pi I}{\pi} \frac{x}{r^{4}}\left[(1+k) x^{2}+(1-k) y^{2}\right], \\
& T_{12} \sim-\frac{\Pi}{\pi} \frac{y}{r^{4}}\left[(1+k) x^{2}+(1-k) y^{2}\right], \\
& T_{22} \sim \frac{\Pi}{\pi} \frac{x}{r^{4}}\left[(1-k) x^{2}+(1-3 k) y^{2}\right] .
\end{aligned}
$$

Case B

$$
T_{11} \sim-\frac{2 \Sigma k}{\pi} \cdot \frac{x^{2} y}{r^{4}}, \quad T_{12} \sim-\frac{2 \Sigma k}{\pi} \frac{x y^{2}}{r^{4}}, \quad T_{22} \sim-\frac{2 \Sigma k}{\pi} \cdot \frac{y^{3}}{r^{4}} .
$$

The singularities in the hyper-stresses can be calculated in the same way from Eqs. (18)-(20) and the corresponding equations for the other components. In plane strain there are ten different hyper-stresses so that it is actually simpler to work out the behaviour of the six strain gradients. It turns out that all of these are just logarithmically singular at the origin (more precisely, $e_{\kappa \lambda, \mu} \sim$ const. $\ln r$ as $r \rightarrow 0$ ).

Taking the asymptotic forms of the displacements in Eqs. (36) and using Eqs. (43) for the coefficients, we readily see that the terms of order $s^{-1}$ in $\bar{u}_{1}$ and $\bar{u}_{2}$ cancel out. The remaining terms, of orders $s^{-3}, x s^{0}, x s^{-2}$, etc., all lead to displacements which are bounded as $r \rightarrow 0$. It is even more remarkable that the strains, $e_{\alpha \beta}$, are also bounded as $r \rightarrow 0$. (The simplest way to see this is to show that the terms of order $x s^{0}$ in $\bar{u}_{1}$ and $\bar{u}_{2}$ are also zero.) Because of these results, the strain energy density (6) is singular only as $(\ln r)^{2}$ as $r \rightarrow 0$, so that the total energy in a region around the singularity is finite.

In the next section we shall compare these results with those obtained previously by other authors.

6. Special cases. The materials considered by Mindlin and Tiersten [3] and by Muki and Sternberg [8] are a special case of second-grade materials for which [6] $P=R=T=l_{1}^{2}=0$ and $Q=\eta$ in the notation of these authors. For such materials condition (42) is not satisfied and the singular forms (47) and (48) are invalid. Since $l_{1}^{2}=0$, the expression $\left(12_{1}\right)$ for $\varphi$ in terms of $\Phi$ is of lower order than the general case, 
and in the solution (25) there will be no $a_{2}$-term. Then in the boundary conditions (29)-(32), the first reduces to an identity, leaving three equations for $\left(a_{1}+i b_{1}\right), i b_{2}$ and $A$. Proceeding as before to derive the asymptotic forms of these coefficients, we obtain Eqs. (38)-(41) with $a=0$ and the above special values for the constants. The solution analogous to Eqs. (43) is

$$
\begin{gathered}
b=A_{1} / \mu, \quad a=0, \quad \gamma=-\eta A_{1} / \mu^{2}, \\
A_{1}=-\frac{\lambda+2 \mu}{2 \lambda+3 \mu}\left(\bar{T}_{1}+i \bar{T}_{2}\right) .
\end{gathered}
$$

Then from Eqs. (33)-(35) we obtain the asymptotic stress transforms, which have the same form as those in Eqs. (44), (45) except that $k$ has the value

$$
k=\frac{\lambda+\mu}{2 \lambda+3 \mu}=(3-2 \nu)^{-1}
$$

where $\nu$ is Poisson's ratio. Then Eqs. (47) and (48) again give the form of the stress singularity.

These results cannot be compared directly with those of Muki and Sternberg, since the two stress tensors are different. For example the symmetric part of these authors' stress tensor is

$$
\tau_{(\kappa \lambda)}=\frac{\partial W}{\partial e_{\kappa \lambda}}=\lambda \Delta \delta_{\kappa \lambda}+\mu\left(u_{\kappa, \lambda}+u_{\lambda, \kappa}\right) .
$$

By calculating the singularities of these stresses in the same way as above it is possible to retrieve Muki and Sternberg's results. It is worth remarking that, for the general case of second-grade materials, these $\tau_{(x \lambda)}$ stresses are actually not singular. This result is tied in with the boundedness of the displacement and strain fields in the second-grade case, in contrast to the Mindlin-Tiersten theory for which the displacements are logarithmically singular and the strains of order $r^{-1}$ as $r \rightarrow 0$.

An even more special case is classical elasticity, which corresponds to taking all the quantities $\left\{F_{k}\right\}$ equal to zero. In this case we must take both $a_{2}$ and $b_{2}$ zero in Eqs. (25) and (26). All of the hyperstresses are identically zero and the boundary conditions (31) and (32) reduce to

$$
\left(a_{1}+i b_{1}\right)=-\frac{\bar{T}_{1}}{2 \mu s^{2}} ; \quad-\frac{\lambda+\mu}{\lambda+2 \mu} A(s)=\bar{T}_{1}+i \bar{T}_{2} .
$$

Proceeding as before, we obtain the same singularities as in Eqs. (47), (48) but with $k=1$. These duplicate the standard results for classical elastic bodies.

The strength of the stress singularity is the same for all three cases, but its form is different, depending on the value of $k$. It is remarkable that the detail of the singularity depends on a single constant, particularly for the general second-grade case where there are five dimensionless ratios of elastic constants on which it could depend. The logarithmic singularities in hyperstress, however, do seem to involve more of these ratios.

Finally we note that in comparing different sets of results for these materials it is necessary to ensure that they refer to the same stress tensor. In particular, as is clear from the boundary condition (2), the stress $T_{\alpha \beta}$ does not describe the forces acting across surface elements within the body. For plane surface elements this force is given by the 
stress $T_{\alpha \beta}-D_{\gamma} M_{\alpha \gamma \beta}$, while in general the action across an element depends on its curvature.

7. Discontinuous loads and double forces. As an idealised case of a load which has a discontinuity at $x=0$ we consider the following two cases.

$$
\begin{array}{rlrlrl}
\text { Case A } & T_{1}(y)=\Pi & (0<y<a), & T_{2}(y)=0 . \\
& =0 & & \text { otherwise. } & & \\
\text { Case B } & T_{1}(y)=0, & T_{2}(y)=\Sigma & (0<y<a), \\
& & & =0 & \text { otherwise. }
\end{array}
$$

The transforms are:

where

$$
A: \bar{T}_{1}(s)=\Pi g(s) ; \quad B: \bar{T}_{2}(s)=\Sigma g(s),
$$

$$
g(s)=\frac{(\sin (s a))}{s}+i \frac{(1-\cos (s a))}{s} .
$$

Eqs. (44) and (45) will still give the singular terms in the stresses. Substituting the above transforms and using the inversion formula (23) leads to the results:

Case A. $T_{11}$ and $T_{22}$ are bounded, $\quad T_{12} \sim \frac{\Pi(1-k)}{\pi} \ln r \quad$ as $\quad r \rightarrow 0$.

Case B. $T_{11}$ and $T_{22}$ are bounded, $\quad T_{22} \sim \frac{2 k \Sigma}{\pi} \ln r$ as $r \rightarrow 0$.

In obtaining these we have used the fact [8] that the expressions $\int_{0}^{\infty} s^{-1} \sin s y e^{-s x} d s$ and $\int_{1}^{\infty} s^{-1} \cos s y e^{-s x} d s+\ln r$ are bounded as $r \rightarrow 0$ as well as Eqs. (46).

The same results with appropriate values for $k$ apply for Mindlin-Tiersten materials and classical elastic materials. It is especially noteworthy that in the classical case, a discontinuity in normal loading does not lead to any singularity whereas the two couplestress theories do lead to a singularity in shear stress. In classical theory, the shear traction boundary condition prevents any singularity, whereas in the present case, it can be seen from Eq. $\left(27_{2}\right)$ that $T_{12}$ and $M_{221}$ can have compensating singularities.

Moving in the direction of greater singularity we can consider a double force, which is the limit of the tractions:

$$
\begin{aligned}
T_{1}(y) & =F & & \text { for } \quad 0<x<a, \\
& =-F & & \text { for } \quad-a<x<0 \\
& =0 & & \text { otherwise }
\end{aligned}
$$

as $a \rightarrow 0$ in such a way that $F a^{2}=C$ remains fixed. The transform is

$$
\overline{T_{1}(s)}=i C s \text {. }
$$

Again Eq. (44) gives the leading terms of the singularity, and making use of the results that

$$
\int_{0}^{\infty} s^{2} e^{-s x} \cos s y d s=\frac{2 x\left(x^{2}-3 y^{2}\right)}{r^{6}} \text { and } \int_{0}^{\infty} s^{2} e^{-s x} \sin s y=\frac{2 y\left(3 x^{2}-y^{2}\right)}{r^{6}}
$$


we obtain that as $r \rightarrow 0$

$$
\begin{aligned}
& T_{11} \sim-\frac{2 C}{\pi} \frac{x y}{r^{6}}\left\{(3 k+1) x^{2}+(1-3 k) y^{2}\right\} \\
& T_{22} \sim \frac{2 C}{\pi} \frac{x y}{r^{6}}\left\{(1+k) x^{2}+(1-3 k) y^{2}\right\} \\
& T_{12} \sim \frac{C}{\pi r^{6}}\left\{(1+k) x^{4}-6 k x^{2} y^{2}-(1-k) y^{4}\right\} .
\end{aligned}
$$

In this case these are only the most singular parts and there will in general be additional logarithmically singular terms.

For the corresponding double shear force we take $\bar{T}_{2}(s)=i C s$ and $\bar{T}_{1}(s)=0$, and this time we get, as $r \rightarrow 0$

$$
\begin{aligned}
& T_{11} \sim \frac{2 k C}{\pi} \frac{x^{2}\left(x^{2}-3 y^{2}\right)}{r^{6}}, \\
& T_{22} \sim \frac{2 k C}{\pi} \frac{y^{2}\left(y^{2}-3 x^{2}\right)}{r^{6}}, \\
& T_{12} \sim \frac{4 k C}{\pi} \frac{x y\left(x^{2}-y^{2}\right)}{r^{6}} .
\end{aligned}
$$

\section{REFERENCES}

1. G. Grioli, Ann. Mat. Pura Appl. 50, 389 (1960)

2. R. A. Toupin, Arch. Rat. Mech. and Anal. 11, 385 (1962)

3. R. D. Mindlin and H. F. Tiersten, Arch. Rat. Mech. and Anal. 11, 415 (1962)

4. W. T. Koiter, Nederl. Akad. Wetensh Ser. Proc. B 67, 17 and 30 (1964)

5. R. A. Toupin, Arch. Rat. Mech. Anal. 17, 85 (1964)

6. R. W. Lardner, Quart. Appl. Math 27, 323 (1969)

7. R. D. Mindlin, Experimental Mechanics 1 (1963)

8. R. Muki and E. Sternberg, Z. Angew. Math. Phys. 16, 611 (1965) 